\title{
Characterization of magnetization-induced second harmonic generation in iron oxide polymer nanocomposites
}

\author{
Stefaan Vandendriessche, ${ }^{*}$ Ventsislav K. Valev, and Thierry Verbiest \\ Molecular Electronics and Photonics, INPAC, K. U. Leuven, Celestijnenlaan 200D, B-3001 Leuven, Belgium \\ *Corresponding author: stefaan.vandendriessche@fys.kuleuven.be \\ Received 22 June 2011; revised 17 August 2011; accepted 17 August 2011; \\ posted 18 August 2011 (Doc. ID 149715); published 9 January 2012
}

\begin{abstract}
We have measured the magnetization-induced second harmonic generation (MSHG) of a nanocomposite consisting of iron oxide nanoparticles in a polymer film. The existing theoretical framework is extended to include DC magnetic fields in order to characterize the MSHG signal and analyze the measurements. Additionally, magnetic hysteresis loops are measured for four principal polarizer-analyzer configurations, revealing the $P_{\mathrm{IN}}-P_{\mathrm{OUT}}$ and $S_{\mathrm{IN}}-P_{\text {OUT }}$ polarizer-analyzer configurations to be sensitive to the transverse magnetic field. These results demonstrate the use of MSHG and the applied formalism as a tool to study magnetic nanoparticles and their magnetic properties. (C) 2012 Optical Society of America OCIS codes: $\quad 160.3820,190.3270,190.4400,310.5448$.
\end{abstract}

\section{Introduction}

Magnetic nanoparticles are the subject of intense investigation in a wide range of disciplines including catalysis, analytical chemistry [1], data storage [2], biochemical research [3], and biomedical applications [4]. The magnetic properties of the nanoparticles play a key role in their applications and can be characterized by a variety of techniques, such as vibrating sample magnetometry [5], Mössbauer spectroscopy, and the superconducting quantum interference device [6]. These techniques provide precise information on the magnetic properties of the nanoparticles but suffer from drawbacks such as high complexity and cost.

Optical second harmonic generation (SHG) is a nonlinear optical process with a large sensitivity to symmetry breaking and interfaces [7]. Because of this sensitivity and the ability to measure buried interfaces, SHG has been used extensively to study interfaces and nanomaterials of various types [8] such as semiconductors [9], plasmonic metal nanoparticles [10], and organic materials [11,12].

1559-128X/12/020209-05\$15.00/0

(C) 2012 Optical Society of America
The presence of a magnetic field does not change the symmetry dependency of SHG, but it does break the time reversal symmetry, allowing for an additional source of SHG: magnetization-induced second harmonic generation (MSHG) [13-17]. This magnetic sensitivity allows the characterization of continuous [18] as well as nanopatterned magnetic interfaces and materials [19].

Besides nanopatterned samples and magnetic films, a variety of magnetic nanoparticle films have been investigated by MSHG [20,21]. These studies have been performed on samples made by techniques such as electron beam lithography, sputtering, and vapor deposition. While these techniques allow for a high degree of control, they can be expensive and time consuming and are not easily accessible for many researchers. Polymer nanocomposites are more accessible materials that allow for improved processability [22]. A wide variety of magnetic nanocomposites exist, and there is currently extensive research on their properties and applications [23].

Although diffuse and depolarized MSHG has been observed from chemically synthesized nanoparticles deposited on a glass substrate through solvent evaporation [24], to our knowledge no characterization 
of the MSHG response of a magnetic polymer nanocomposite has been performed yet. In this paper we report the characterization and analysis of the MSHG response of an iron oxide nanoparticle polymer nanocomposite. These magnetic nanocomposites provide a model system to study the magnetic properties of nanoparticles. In combination with the high sensitivity of MSHG, this knowledge offers the prospect of MSHG measurements in more complex samples containing magnetic nanoparticles.

\section{Experiments}

Iron oxide nanoparticles were synthesized by heating a round-bottom flask with $37.5 \mathrm{ml}$ of diethyleneglycol and $25 \mathrm{ml}$ of octylamine to $150^{\circ} \mathrm{C}$ while stirring [25]. Separately, $2.4 \mathrm{~g}$ of anhydrous $\mathrm{FeCl}_{3}$ was dissolved in a beaker with $10 \mathrm{ml}$ of diethyleneglycol and $3.5 \mathrm{ml}$ of water. This mixture was added to the round-bottom flask, which was subsequently refluxed at $180^{\circ} \mathrm{C}$ for 24 hours. The synthesized nanoparticles were filtered on a homemade magnet, washed three times with acetone, and dried in a vacuum oven. This resulted in iron oxide nanoparticles with a diameter of $7.63 \mathrm{~nm} \pm 2.09 \mathrm{~nm}$ as determined by transmission electron microscopy.

The nanocomposite was synthesized by sonicating $38 \mathrm{mg}$ of the iron oxide nanoparticles with $226 \mathrm{mg}$ of poly(methyl) methacrylate (PMMA) with a nominal molecular weight of 38,000 in $2 \mathrm{ml}$ of chloroform for $1 \mathrm{~h}$. This solution was spin coated on a glass plate at $1800 \mathrm{rpm}$ for $15 \mathrm{~s}$, resulting in a film of approximately $2.1 \mu \mathrm{m}$ thick.

SHG was measured using a Ti:sapphire laser operating at a wavelength of $800 \mathrm{~nm}$, emitting approximately $100 \mathrm{fs}$ pulses with a repetition rate of $82 \mathrm{MHz}$. The generated beam was polarized by a half-wave plate on a motorized rotation stage followed by a Glan-laser polarizer mounted on a separate motorized rotation stage. Subsequently, the beam passed through a quarter-wave plate, which was also mounted on a motorized rotation stage. After being filtered by an RG 650 filter to exclude $400 \mathrm{~nm}$ light, the beam of approximately $100 \mathrm{~mW}$ intensity was focussed (focal length $10 \mathrm{~cm}$ ) on the sample at an incidence angle of $45^{\circ}$. The sample was mounted in a GMW 3470 dipole electromagnet with the magnetic field in a transverse configuration. The transmitted beam was filtered by two BG 39 filters to exclude $800 \mathrm{~nm}$ light, allowing the $\mathrm{SH}$ light to pass through an analyzer mounted on a motorized rotation stage. A photomultiplier tube cooled to $-20^{\circ} \mathrm{C}$ collected the second harmonic light, and after preamplification, an SR400 gated photon counter was used to process the signal.

\section{Results and Discussion}

Within the electric dipole approximation, the optical polarization induced at the second harmonic frequency can be written as [7]

$$
P_{i}(2 \omega)=\sum_{j, k} \chi_{i j k}^{(2)} E_{j}(\omega) E_{k}(\omega)
$$

where $i, j$ and $k$ are the Cartesian axes, $E_{i}$ is the electric field of the incident light, and $\chi^{(2)}$ is the secondorder susceptibility, a material-specific second-rank tensor consisting of 27 components. The presence of a magnetic field allows for additional sources of SHG [14]:

$$
P_{i}(2 \omega)=\sum_{j, k} \chi_{i j k}^{(2)} E_{j}(\omega) E_{k}(\omega)+\sum_{j, k, l} \chi_{i j k l}^{(3)} E_{j}(\omega) E_{k}(\omega) M_{l},
$$

where $M_{l}$ is the magnetization in the $l$ direction and $\chi^{(3)}$ is the third-order magnetic susceptibility, a material-specific third-rank tensor consisting of 81 components.

The MSHG from a $14.4 \mathrm{wt} . \%$ iron oxide nanoparticle PMMA nanocomposite of approximately $2.1 \mu \mathrm{m}$ thick was measured in transmission at an incidence angle of $45^{\circ}$ in a transverse magnetic field, as depicted in Fig. 1.

We analyzed the dependence of the MSHG on the polarization of the incident light and on the sign of the magnetic field (Fig. 2). A clear influence of the sign of the transverse magnetic field on the intensity of the SHG signal is seen in all four principal polarizeranalyzer configurations. Regardless of symmetry and experimental geometry, it is always possible to describe the intensity of the (M)SHG with Eq. (3) [7]

$$
I(2 \omega)_{s, p} \sim\left[f_{s, p} E_{p}^{2}(\omega)+g_{s, p} E_{s}^{2}(\omega)+h_{s, p} E_{p}(\omega) E_{s}(\omega)\right]^{2},
$$

where $E_{s}, E_{p}$ are, respectively, the $s$ and $p$ components of the electric field of the incident light and $I(2 \omega)_{s, p}$ is the intensity of, respectively, the $s$ and $p$ components of the SHG.

Based on symmetry considerations within the electric dipole approximation for an isotropic sample with interfaces with $C_{v}^{\infty}$ symmetry, the theoretical expressions for the $f, g$, and $h$ parameters of an in-plane isotropic sample in transmission at $45^{\circ}$ can be derived to be [7]

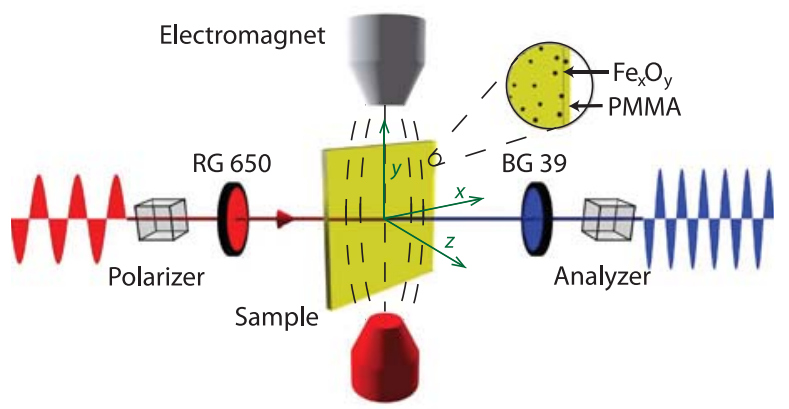

Fig. 1. (Color online) Experimental setup for MSHG measurements of an iron oxide nanocomposite. A transverse magnetic field is applied, and the sample is measured at $45^{\circ}$ in transmission. 


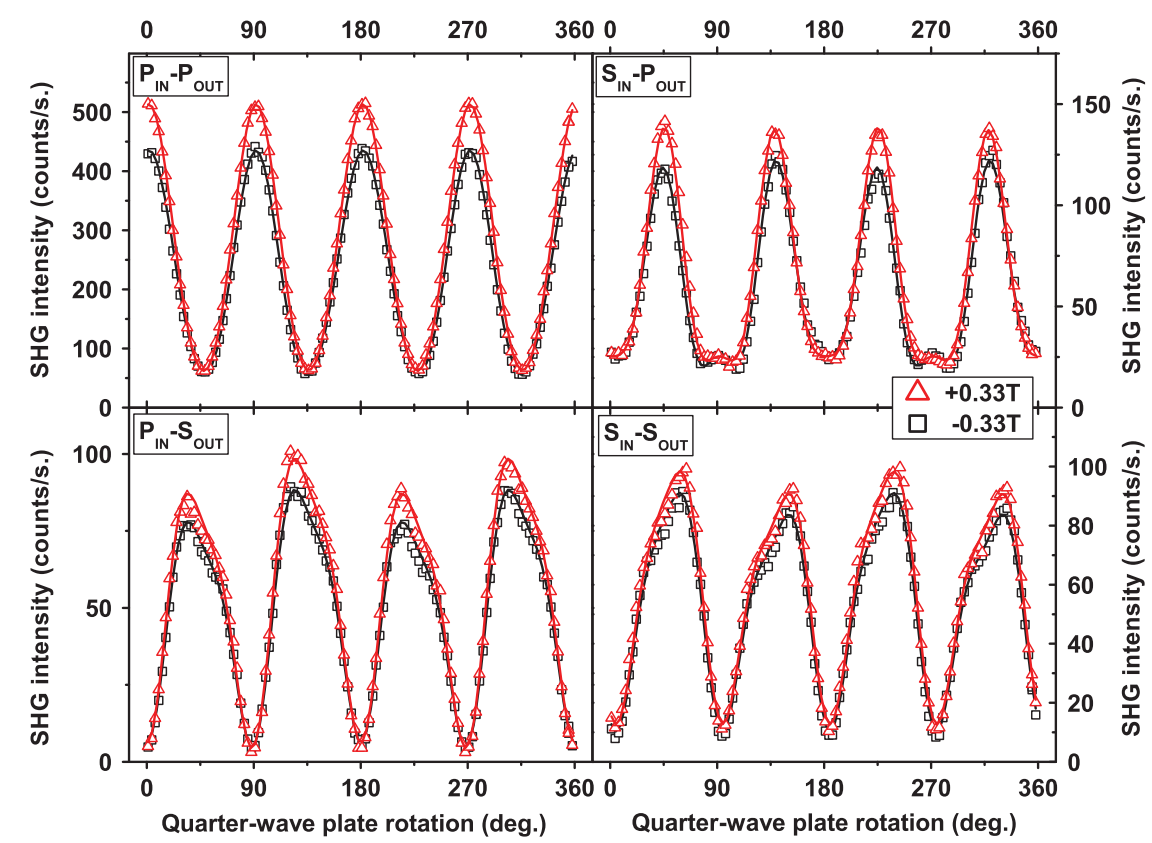

Fig. 2. (Color online) Magnetic contrast of the SHG intensity for four principal polarizer-analyzer configurations. Measurements performed as a function of the input polarization by rotating a quarter-wave plate placed before the sample. Solid lines are the fits according to Eq. (3), and the fitting parameters are displayed in Table 1. A clear influence of the sign of the magnetic field on the intensity of the SHG signal is seen in all four principal polarizer-analyzer configurations.

$$
\begin{gathered}
f_{s}=0 \\
g_{s}=0 \\
h_{s}=\sqrt{2}\left(\chi_{y y z}^{(2)}+\chi_{y x y y}^{(3)} M_{y}\right) \\
f_{p}=\frac{1}{(\sqrt{2})^{3}}\left(\chi_{z x x}^{(2)}+\chi_{z z z}^{(2)}+2 \chi_{x x z}^{(2)}\right. \\
\left.+\left(\chi_{x x x y}^{(3)}+\chi_{x z z y}^{(3)}+2 \chi_{z x z y}^{(3)}\right) M_{y}\right) \\
g_{p}=\frac{1}{\sqrt{2}}\left(\chi_{z x x}^{(2)}+\chi_{x y y y}^{(3)} M_{y}\right) \\
h_{p}=0 .
\end{gathered}
$$

Note that we have omitted the linear optical coefficients for simplicity, though these can be included in a straightforward fashion [26]. Equation (3) can be adapted to allow for a rotating wave plate in front of the sample [27]. Our experimental data were fitted to this equation. The fitting parameters for the fit in Fig. 2 are displayed in Table 1. Within experimental precision the fitting parameters $f_{p}, g_{p}$, and $h_{s}$ are significantly influenced by the transverse magnetic field, which corresponds well to the theoretical predications from Eqs. (4)-(9). The largest magnetic contribution originates from the $\left(\chi_{x x x y}^{(3)}+\chi_{x z z y}^{(3)}+2 \chi_{z x z y}^{(3)}\right)$ components of the second order magneto-susceptibility.

The small deviation of the components from those predicted by the model is attributable either to bulk contributions not included in the model, or to anisotropy induced by sample preparation [25]. In the case of samples with a known anisotropy, it is possible to derive Eqs. (4)-(9) for the appropriate symmetry, yielding the tensor component values for the directional (magnetic) anisotropy present in these samples. Here the possible sample preparation induced anisotropy is assumed to be small; thus, the deviation from 0 for the $f_{s}$ and $g_{s}$ components is primarily attributable to bulk contributions.

Table 1. Fitting Components of the Fit of Eq. (3) to the Data in Fig. $2^{a}$

\begin{tabular}{ccc}
\hline & $+0.33 \mathrm{~T}$ & $-0.33 \mathrm{~T}$ \\
\hline$f_{s}$ & $13.223+4.196 i \pm(0.370+0.273 i)$ & $11.987+3.918 i \pm(0.358+0.257 i)$ \\
$g_{s}$ & $14.002-0.297 i \pm(0.388+0.371 i)$ & $12.556-0.538 i \pm(0.359+0.345 i)$ \\
$h_{s}$ & $140.773 \pm 0.495$ & $132.904 \pm 0.481$ \\
$f_{p}$ & $-6.821+186.594 i \pm(2.247+0.433 i)$ & $-6.459+170.265 i \pm(2.800+0.494 i)$ \\
$g_{p}$ & $-7.682+22.944 i \pm(0.286+1.618 i)$ & $-13.382+22.660 i \pm(0.371+1.989 i)$ \\
$h_{p}$ & $0.383 \pm 0.180$ & $-1.190 \pm 0.228$ \\
\hline
\end{tabular}

${ }^{a}$ Within experimental precision, $h_{s}, f_{p}$, and $g_{p}$ are significantly influenced by the transverse magnetic field, corresponding well to the theoretical expressions Eqs. (4)-(9). 


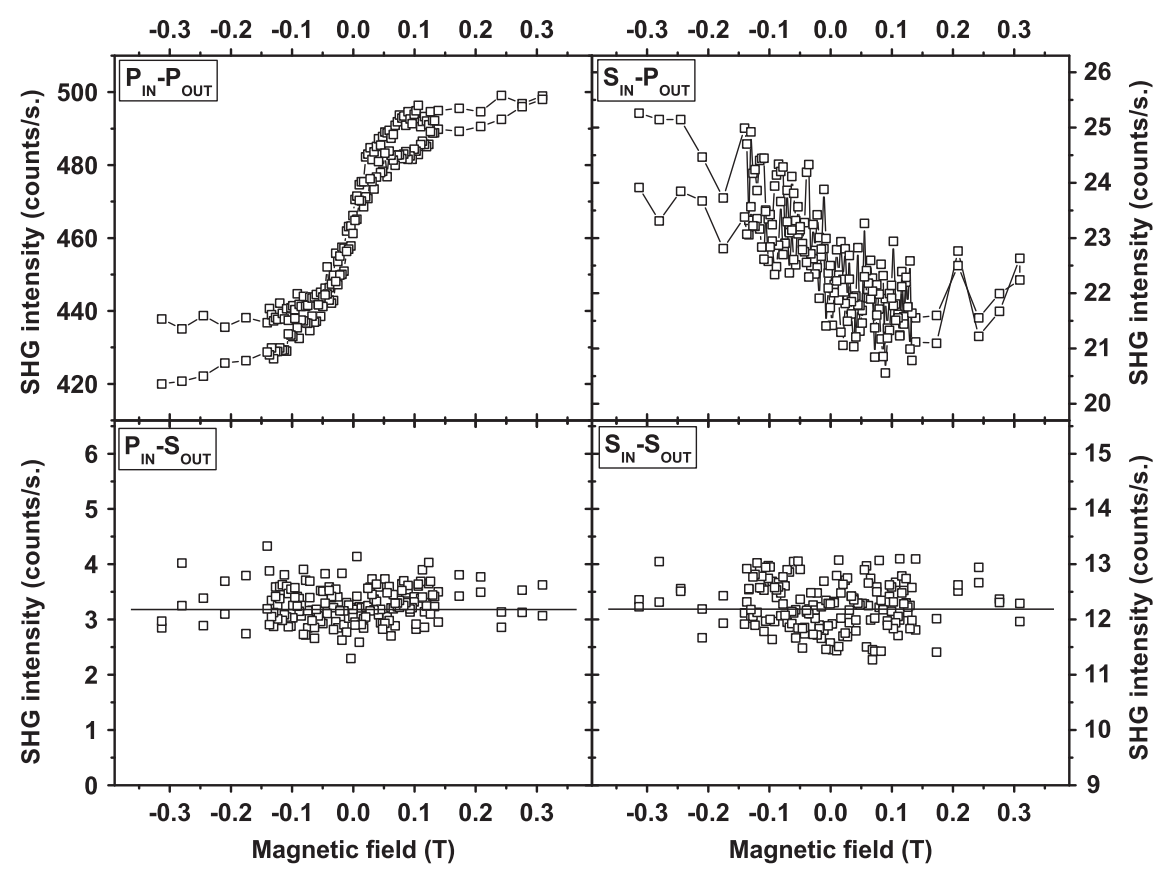

Fig. 4. MSHG hysteresis loops for four principal polarizer-analyzer configurations. $P_{\mathrm{IN}}-P_{\mathrm{OUT}}$ and $S_{\mathrm{IN}}-P_{\mathrm{OUT}}$ SHG is sensitive to the transverse magnetic field, with relative changes in SHG of more than $10 \% . P_{\mathrm{IN}-} S_{\text {OUT }}$ and $S_{\mathrm{IN}}-S_{\text {OUT }}$ show no magnetic contrast.

Subsequently, the polarization of the MSHG signal was verified by rotating the analyzer for both $p$ - and $s$-polarized incident light $\left(P_{\mathrm{IN}}\right.$ and $\left.S_{\mathrm{IN}}\right)$ (Fig. 3). For the $P_{\mathrm{IN}}$ configuration, the rotation of the analyzer results in a transition of the measurement of the parameter $f_{p}$ to the parameter $f_{s}$. A large magnetic contrast in intensity but no clear polarization rotation is observed, in agreement with the large magnetic contrast for the $f_{p}$ parameter and a smaller magnetic contrast and absolute value of the $f_{s}$ para-

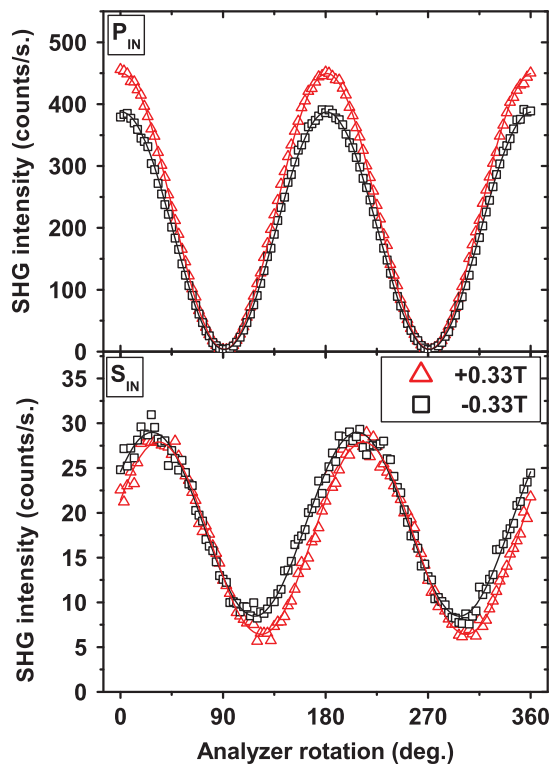

Fig. 3. (Color online) Polarization of the MSHG, measured by rotation of the analyzer, where $0^{\circ}$ analyzer rotation corresponds to $P_{\text {OUT }}$. The SHG generated by $P_{\text {IN }}$ polarized light mainly varies in amplitude upon reversal of the sign of the magnetic field, while the SHG generated by $S_{\text {IN }}$ polarized light slightly rotates the polarization upon reversal of the magnetic field. meter. For the $S_{\text {IN }}$ configuration, the analyzer rotation results in a transition of the measurement of the parameter $g_{p}$ to the parameter $g_{s}$. A smaller magnetic contrast in intensity is observed, and there is a small rotation of polarization due to the magnetic field. This corresponds to a small interference between the bulk $g_{s}$ component and the magnetic $g_{p}$ component, and the small size of the rotation illustrates the significance of the magnetic contribution.

MSHG hysteresis loops were measured for four principal polarizer-analyzer configurations. These loops can accurately represent the magnetization reversal process for systems where the magnetic contrast is small in comparison with the overall SHG response, as is the case here (Fig. 4) [28]. In the $P_{\mathrm{IN}}-P_{\mathrm{OUT}}$ and $S_{\mathrm{IN}}-P_{\mathrm{OUT}}$ configurations, the magnetic contrast of, respectively, the $f_{p}$ and the $g_{p}$ parameter is measured. The resulting magnetic contrast corresponds well with the obtained fitting parameters, showing the largest contribution to the magnetic susceptibility to be from the $\left(\chi_{x x x y}^{(3)}+\chi_{x z z y}^{(3)}+2 \chi_{z x z y}^{(3)}\right)$ term. In the $P_{\text {IN }}-S_{\text {OUT }}$ and $S_{\text {IN }}-S_{\text {OUT }}$ configurations, the magnetic contrast of, respectively, the $f_{s}$ and the $g_{s}$ term is measured. The lack of magnetic contrast indicates that, consistent with the fitting parameters, the $f_{s}$ and $g_{s}$ terms are not influenced by a magnetic field within experimental precision.

\section{Conclusions}

The MSHG measurements are consistent with each other, and the theoretical model provides a good framework to analyze the MSHG of nanocomposites. The obtained fitting parameters yield more insight into the nonlinear susceptibilities of the nanocomposite and its nanoparticles than the magnetic contrast, which is represented by a single number. 
Additionally, the theoretical parameters can be derived for an arbitrary symmetry, making it possible to identify directional (magnetic) anisotropy in more complex samples. To determine the components of the (magnetic) nonlinear susceptibilities, only a single measurement at one incidence angle in either transmission or reflection is required. A further advantage is that characterization is possible with a wave plate of any retardation besides a half-wave plate, not only a quarter-wave plate [27].

These data show magnetic nanocomposites to be promising materials for MSHG investigation. Further nanocomposite MSHG measurements will allow a better understanding of the influence of magnetic nanoparticle parameters, e.g., size, on their nonlinear susceptibilities. This knowledge can support future applications for magnetic nanoparticles such as allowing the identification of magnetic nanoparticles in SHG microscopy images.

In conclusion, we have measured and characterized the MSHG response of an iron oxide nanoparticle polymer nanocomposite. Experimental data show a large magnetic contrast of SHG in a transverse magnetic field. Coupling this data to a theoretical framework allows the measurements to be correlated to the nonlinear (magnetic) polarizabilities of the nanocomposite. This theoretical understanding demonstrates the usefulness of MSHG to characterize the magnetic properties of nanoparticles.

S. V. and V. K. V. are grateful for the financial support from the FWO-Vlaanderen. We are grateful to the University of Leuven (GOA) for financial support.

\section{References}

1. J. S. Beveridge, J. R. Stephens, and M. E. Williams, "The use of magnetic nanoparticles in analytical chemistry," Annu. Rev. Anal. Chem. 4, 251-273 (2010).

2. A.-H. Lu, E. L. Salabas, and F. Schüth, "Magnetic nanoparticles: synthesis, protection, functionalization, and application," Angew. Chem. Int. Ed. 46, 1222-1244 (2007).

3. E. Katz and I. Willner, "Integrated nanoparticle-biomolecule hybrid systems: synthesis, properties, and applications," Angew. Chem. Int. Ed. 43, 6042-6108 (2004).

4. A. K. Gupta and M. Gupta, "Synthesis and surface engineering of iron oxide nanoparticles for biomedical applications," Biomaterials 26, 3995-4021 (2005).

5. S. Foner, "Versatile and sensitive vibrating sample magnetometer," Rev. Sci. Instrum. 30, 548-557 (1959).

6. G. F. Goya, T. S. Berquo, F. C. Fonseca, and M. P. Morales, "Static and dynamic magnetic properties of spherical magnetite nanoparticles," J. Appl. Phys. 94, 3520-3528 (2003).

7. T. Verbiest, K. Clays, and V. Rodriguez, Second-Order Nonlinear Optical Characterization Techniques: An Introduction (CRC Press, 2009).

8. Y. R. Shen, "Surface properties probed by second-harmonic and sum-frequency generation," Nature 337, 519-525 (1989).

9. V. K. Valev, M. K. Vanbel, B. Vincent, V. V. Moshchalkov, M. Caymax, and T. Verbiest, "Second harmonic generation indicates a better $\mathrm{Si} / \mathrm{Ge}$ interface quality for higher temperature and with $\mathrm{N}_{2}$ rather than with $\mathrm{H}_{2}$ as the carrier gas," IEEE Electron. Device Lett. 32, 12-14 (2011).

10. I. V. Kityk, J. Ebothé, I. Fuks-Janczarek, A. A. Umar, K. Kobayashi, M. Oyama, and B. Sahraoui, "Nonlinear optical properties of Au nanoparticles on indium-tin oxide substrate," Nanotechnology 16, 1687-1692 (2005).

11. M. M. Kauranen, T. Verbiest, A. Persoons, E. W. Meijer, M. N. Teerenstra, A. J. Schouten, R. J. M. Nolte, and E. E. Havinga, "Chiral effects in the second-order optical nonlinearity of a poly(isocyanide) monolayer," Adv. Mater. 7, 641-644 (1995).

12. M. Kauranen, S. V. Elshocht, T. Verbiest, and A. Persoons, "Tensor analysis of the second-order nonlinear optical susceptibility of chiral anisotropic thin films," J. Chem. Phys. 112, 1497-1502 (2000).

13. P. S. Pershan, "Nonlinear optical properties of solids: energy considerations," Phys. Rev. 130, 919-929 (1963).

14. R. P. Pan, H. D. Wei, and Y. R. Shen, "Optical second-harmonic generation from magnetized surfaces," Phys. Rev. B 39, 1229 1234 (1989).

15. J. Reif, J. C. Zink, C. M. Schneider, and J. Kirschner, "Effects of surface magnetism on optical second harmonic generation," Phys. Rev. Lett. 67, 2878-2881 (1991).

16. A. Kirilyuk and T. Rasing, "Magnetization-induced-secondharmonic generation from surfaces and interfaces," J. Opt. Soc. Am. B 22, 148-167 (2005).

17. J. F. McGilp, L. Carroll, K. Fleischer, J. P. Cunniffe, and S. Ryan, "Magnetic second-harmonic generation from interfaces and nanostructures," J. Magn. Magn. Mater. 322, 1488-1493 (2010).

18. V. K. Valev, M. Gruyters, A. Kirilyuk, and T. Rasing, "Direct observation of exchange bias related uncompensated spins at the $\mathrm{CoO} / \mathrm{Cu}$ interface," Phys. Rev. Lett. 96, 067206 (2006).

19. V. K. Valev, A. V. Silhanek, W. Gillijns, Y. Jeyaram, H. Paddubrouskaya, A. Volodin, C. G. Biris, N. C. Panoiu, B. De Clercq, M. Ameloot, O. A. Aktsipetrov, V. V. Moshchalkov, and T. Verbiest, "Plasmons reveal the direction of magnetization in nickel nanostructures," ACS Nano 5, 91-96 (2011).

20. Y. F. Chiang, Y. J. Hsu, T. M. Liu, H. W. Chu, J. G. Lin, C. H. Chen, and Y.-M. Chang, "Magnetization reversal process of ferromagnetic granular thin films probed by magnetizationinduced second harmonic generation," Appl. Phys. Lett. 95, 172515 (2009).

21. O. A. Aktsipetrov, T. V. Murzina, E. M. Kim, R. V. Kapra, A. A. Fedyanin, M. Inoue, A. F. Kravets, S. V. Kuznetsova, M. V. Ivanchenko, and V. G. Lifshits, "Magnetization-induced second- and third-harmonic generation in magnetic thin films and nanoparticles," J. Opt. Soc. Am. B 22, 138-147 (2005).

22. L. L. Beecroft and C. K. Ober, "Nanocomposite materials for optical applications," Chem. Mater. 9, 1302-1317 (1997).

23. S. Behrens, "Preparation of functional magnetic nanocomposites and hybrid materials: recent progress and future directions," Nanoscale 3, 877-892 (2011).

24. I. A. Kolmychek, T. V. Murzina, S. Fourier, J. Wouters, V. K. Valev, T. Verbiest, and O. A. Aktsipetrov, "Second harmonic generation in core (shell) $y$-Fe2O3 (Au) nanoparticles," Solid State Phenom. 152-153, 508-511 (2009).

25. J. Wouters, O. I. Lebedev, G. V. Tendeloo, H. Yamada, N. Sato, J. Vanacken, V. V. Moshchalkov, T. Verbiest, and V. K. Valev, "Preparing polymer films doped with magnetic nanoparticles by spin-coating and melt-processing can induce an in-plane magnetic anisotropy," J. Appl. Phys. 109, 076105 (2011).

26. J. J. Maki, M. Kauranen, and A. Persoons, "Surface secondharmonic generation from chiral materials," Phys. Rev. B 51, 1425-1434 (1995).

27. J. J. Maki, M. Kauranen, T. Verbiest, and A. Persoons, "Uniqueness of wave-plate measurements in determining the tensor components of second-order surface nonlinearities," Phys. Rev. B 55, 5021-5026 (1997).

28. V. K. Valev, M. Gruyters, A. Kirilyuk, and T. Rasing, "Influence of quadratic contributions in magnetization-induced second harmonic generation studies of magnetization reversal," Phys. Status Solidi B 242, 3027-3031 (2005). 